\title{
Early vessel normalization improves glioblastoma outcomes
}

A subset of patients with glioblastoma who respond by showing increased tumour perfusion after early antiangiogenic therapy are more likely to benefit from anti-VEGF treatment combined with radiation and chemotherapy than patients who don't respond. Furthermore, vascular normalization is associated with longer overall survival in patients with hyperpermeable (but uncompressed) tumours, according to two new studies.

\section{An early marker of response was identified... 77}

Rakesh Jain, senior author on both studies, first hypothesized in 2001 that anti-VEGF therapy works by 'normalizing' leaky vessels that surround tumours, improving the delivery of drugs and oxygen, which are also necessary for effective radiotherapy. Drugs that inhibit the VEGF pathway only benefit a subset of patients with solid tumours, and determining biomarkers that can distinguish which patients are more likely to respond to treatment had yet to be identified.

In the first study, the researchers used MRI techniques to measure changes in blood tumour vasculature, including vessel architectural imaging, to monitor changes after anti-VEGF therapy. "Early increased tumour perfusion, often seen after one treatment with a pan-VEGFR kinase inhibitor, was associated with a twothirds increase in survival time in patients harbouring glioblastoma, compared with those patients who had no increase in blood perfusion," explain four authors of the study-Batchelor, Emblem, Duda and Jain-in a joint statement. This effect, seen in half of the patients who underwent anti-VEGF therapy and chemoradiation, was not observed in patients who received chemotherapy alone. Moreover, these changes are associated with improved oxygenation and various plasma biomarkers. "An early marker of response was identified," state the authors, "which may be critical for the use of anti-VEGF drugs in patients with the most lethal form of brain cancer."
In the second study, the authors used a mathematical model to compare the effectiveness of vascular normalization with stress-alleviation treatment strategies across tumour types. Vascular normalization was found to be more effective in tumours with hyperpermeable (but uncompressed) vessels, such as glioblastomas. Stress-alleviation is, conversely, more effective for compressed (but hypopermeable) vessels.

These studies highlight markers that might be able to distinguish patients for whom expensive, and potentially toxic, anti-VEGF therapy will be beneficial, and also provide guidance on choosing normalization or stress-alleviation strategies to treat patients, according to cancer types.

Bryony Jones

Original articles Batchelor, T. T. et al. Improved tumor oxygenation and survival in glioblastoma patients who show increased blood perfusion after cediranib and chemoradiation. Proc. Nat/Acad. Sci. USA doi:10.1073/pnas.1318022110 | Stylianopoulos, T. \& Jain, R. K. Combining two strategies to improve perfusion and drug delivery in solid tumors. Proc. Natl Acad. Sci. USA 110, 18632-18637 (2013) 